\title{
Three-year Clinical Evaluation of Different Restorative Resins in Class I Restorations
}

\author{
AR Yazici • I Ustunkol • G Ozgunaltay \\ B Dayangac
}

\begin{abstract}
Clinical Relevance
Except for the better marginal adaptation that packable resin composite showed, siloranebased restorative, nanofilled resin, and packable resin composite resulted in similar clinical performance in restoring Class I cavities after 3 years.
\end{abstract}

\section{SUMMARY}

The aim of the present study was to evaluate the three-year clinical performance of a nanofilled resin composite, a packable resin composite, and silorane-based resin restorations in Class I occlusal cavities. Twenty-eight patients with at least three similar-sized occlusal lesions in molar teeth participated in the study. A total of 84 Class I occlusal restorations were placed: 28 with nanofilled resin composite (Filtek Supreme), 28 with packable resin com-

*A. Ruya Yazici, DDS, PhD, professor, Department of Restorative Dentistry, School of Dentistry, Hacettepe University, Ankara, Turkey

Ildem Ustunkol, DDS, research assistant, Department of Restorative Dentistry, School of Dentistry, Hacettepe University, Ankara, Turkey

Gul Ozgunaltay, DDS, PhD, professor, Department of Restorative Dentistry, School of Dentistry, Hacettepe University, Ankara, Turkey

Berrin Dayangac, DDS, PhD, professor, Department of Restorative Dentistry, School of Dentistry, Hacettepe University, Ankara, Turkey

*Corresponding author: 06100, Sihhiye, Ankara, Turkey; email: ruyay@hacettepe.edu.tr

DOI: $10.2341 / 13-221-\mathrm{C}$ posite (P60), and 28 with silorane-based resin (Filtek Silorane). Filtek Supreme and $P 60$ were used with their respective etch-and-rinse adhesive system, Adper Single Bond 2, and Filtek Silorane was used with its respective self-etch adhesive, Filtek Silorane Adhesive. All restorations were placed by the same operator. The restorations were evaluated at baseline, at six months, and annually for three years according to modified US Public Health Service criteria by two calibrated examiners who did not know which restorative resin had been used. The three restorative materials for each category were compared using the $\chi^{2}$ test at a significance level of 0.05 . Cochran's $Q$ test was used to compare the changes across the five time points for each restorative material. McNemar's test followed by Bonferroni adjustment was used when significance differences were found. At the end of the three years, 60 restorations were evaluated in 20 patients, with a recall rate of $71.4 \%$. The retention rate was $100 \%$ for all restorative resins. Eight restorations from the $\mathbf{P 6 0}$ group, ten from the Filtek Supreme group, and nine from the Filtek Silorane group were rated Bravo for marginal discoloration. For marginal adapta- 
tion, three P60, five Filtek Supreme, and 11 Filtek Silorane restorations were rated Bravo. No statistically significant differences in overall clinical performance were found between the restorative materials except for marginal adaptation. P60 showed the best marginal adaptation at the end of the three years. No differences were observed between the restorative resins for any of the evaluation criteria tested $(p>0.05)$. None of the restorations showed postoperative sensitivity, secondary caries, or loss of anatomic form. All restorative resins performed equally well in clinical conditions during the three-year evaluation, and no significant differences were found among them, except for marginal adaptation, in which P60 showed superior results.

\section{INTRODUCTION}

Patients' increasing demand for esthetic restorations, even in posterior regions, has led to improvements in the mechanical and physical properties of composite restorative materials. Currently, composite resins have been diversified according to chemical composition and particle filler size, with the objective of finding better ones.

Difficulty in restoration placement and establishment of appropriate proximal contacts as well as the sticky form of conventional composite resins prompted producers to introduce packable or condensable composite resins to overcome these handicaps. ${ }^{1}$ Packable resin composites are claimed to be suitable for stress-bearing posterior restorations. Although packable resins were developed for better clinical performance, some studies showed that the properties of packable composites are not superior to those of conventional resin composites. ${ }^{2-4}$

A new type of resin composite, so-called nanocomposite, has been developed recently to improve the performance of resins. The use of nanotechnology in resin composite systems offers high translucency and high polish, similar to that of microfilled composites, while maintaining physical properties and wear equivalent to several hybrid composites. ${ }^{5}$ However, shrinkage during polymerization is still a major challenge. Different techniques and restoratives have been proposed to overcome this phenomenon. Lowshrinkage silorane-based resins such as Filtek Silorane have been suggested as a means to reduce shrinkage and cuspal deflection. ${ }^{6}$ During siloranebased resin composite polymerization, ring-opening monomers connect by opening, flattening, and extending toward each other. This mechanism results in less volumetric shrinkage compared with methacrylate-based composites. As methacrylate-based composites cure, the molecules of these "linear monomers" connect by actually shifting closer together in a linear response that causes a loss of volume. ${ }^{7}$

Many laboratory studies have addressed bond strength of silorane-based restorative on tooth structure; however, it is of paramount relevance to study the clinical behavior of silorane restorative resin, as information about the clinical performance of this restorative is very limited. ${ }^{8-12}$ Moreover, the results of these in vitro studies cannot always be directly extrapolated to the performance of restorative materials.

The aim of this clinical study was to investigate whether a low-shrinkage silorane-based restorative resin improves the clinical performance of restorations in Class I cavities. We tested the hypothesis that a low-shrinkage silorane-based restorative resin would show better clinical results than the packable and nanofilled resin composites because of its lower polymerization shrinkage.

\section{METHODS AND MATERIALS}

\section{Selection Criteria}

Approval for this clinical study was obtained from the Human Ethics Committee of Hacettepe University. The subjects were recruited from patients seeking routine dental care at the Department of Restorative Dentistry, Hacettepe University.

For inclusion in the study, the teeth to be restored had to be vital and without pulpal or periodontal disease, pain, and preoperative sensitivity. Teeth with an existing occlusal contact and at least one neighboring tooth were required. Patients with poor oral hygiene, serious health problems, heavy bruxism, or a known allergy to the substances used in the study were not included. Before participating in the study, all patients signed a written consent form after receiving a full explanation of the treatment procedure.

Of the 50 patients evaluated, only the 28 (17 women and 11 men) who had three primary carious lesions that required Class I restorations in their molars (first or second molars) were enrolled in the study. The 28 subjects had a mean age of 29.3 (range $=18-52$ years).

\section{Restorative Procedures}

Bitewing radiographs of the teeth to be restored were taken preoperatively. The lesion depth was the 
middle or beyond the middle-third of the dentin. The teeth to be restored were first cleaned with a nonfluoridated prophylaxis paste on a slowly rotating rubber cup and then washed and dried. Isolation was accomplished using cotton rolls. The cavity was prepared using a diamond bur (Diatech, Heerbrugg, Switzerland) with a high-speed handpiece. Carious tissue was removed using a slow-speed steel round bur and excavator. Removal of carious tissue was checked with visual and tactile feedback from an explorer. The teeth to be restored were randomly assigned according to a table of random numbers for restoration with a packable resin composite, P60 (3M, ESPE, St Paul, MN, USA); a nanofilled resin composite, Filtek Supreme (3M); or a low-shrinkage silorane-based restorative resin, Filtek Silorane (3M). Filtek Supreme and P60 were used with their respective etch-and-rinse adhesive system, Adper Single Bond 2 (3M), and Filtek Silorane was used with its respective adhesive, Filtek Silorane Adhesive (3M). Enamel and dentin were etched for 30 and 15 seconds, respectively, with $37 \%$ phosphoric acid gel (3M) for the P60 and Filtek Supreme groups. The adhesive systems were applied strictly according to the manufacturers' instructions (Table 1). The restorative resins were placed in increments not exceeding $2 \mathrm{~mm}$ in thickness, and each increment was cured for 40 seconds with a quartz-tungstenhalogen curing unit (Benlioglu, Ankara, Turkey) at $600 \mathrm{~mW} / \mathrm{cm}^{2}$. Finishing was accomplished using finishing diamond burs (Diatech) at high speed, and polishing was carried out with silicon points and flexible discs (SwissFlex, Diatech). They were all used under constant water cooling. Occlusion was checked with articulating paper and adjusted. Clinical photographs at 1:1 magnification were also taken before and after surgery, at baseline, and at each recall with a digital camera (Canon, PowerShot $300 \mathrm{HS}$, Melville, NY, USA).

\section{Evaluation Criteria and Procedures}

Patients were recalled at baseline (one week after placement), at six months after placement, and at one, two, and three years after placement. Two calibrated examiners, other than the operator, evaluated each restoration. Modified US Public Health Service criteria described by Cvar and Ryge were used to evaluate the following characteristics: retention, marginal discoloration, marginal adaptation, color match, surface texture, anatomic form, and secondary caries (Table 2). ${ }^{13}$ Postoperative sensitivity was assessed by air and/or tactile contact and was recorded as absent, mild, or severe. Sensitivity to air was assessed by blowing a stream of compressed air for five seconds while shielding the neighboring teeth with the fingers. Sensitivity to tactile contact was assessed by moving a probe over the restored tooth surface. Subjects were also questioned regarding sensitivity to cold/hot or other stimuli.

The examiners were calibrated to a predetermined level of inter- and intraexaminer agreement of at least $95 \%$ per criterion. The calibrated examiners were blinded to the restorative material used. Any discrepancy in evaluation between the two evaluators was immediately resolved at chair-side. The restorations were scored as follows: Alpha represented the ideal clinical situation, Bravo was clinically acceptable, and Charlie represented a clinically unacceptable situation.

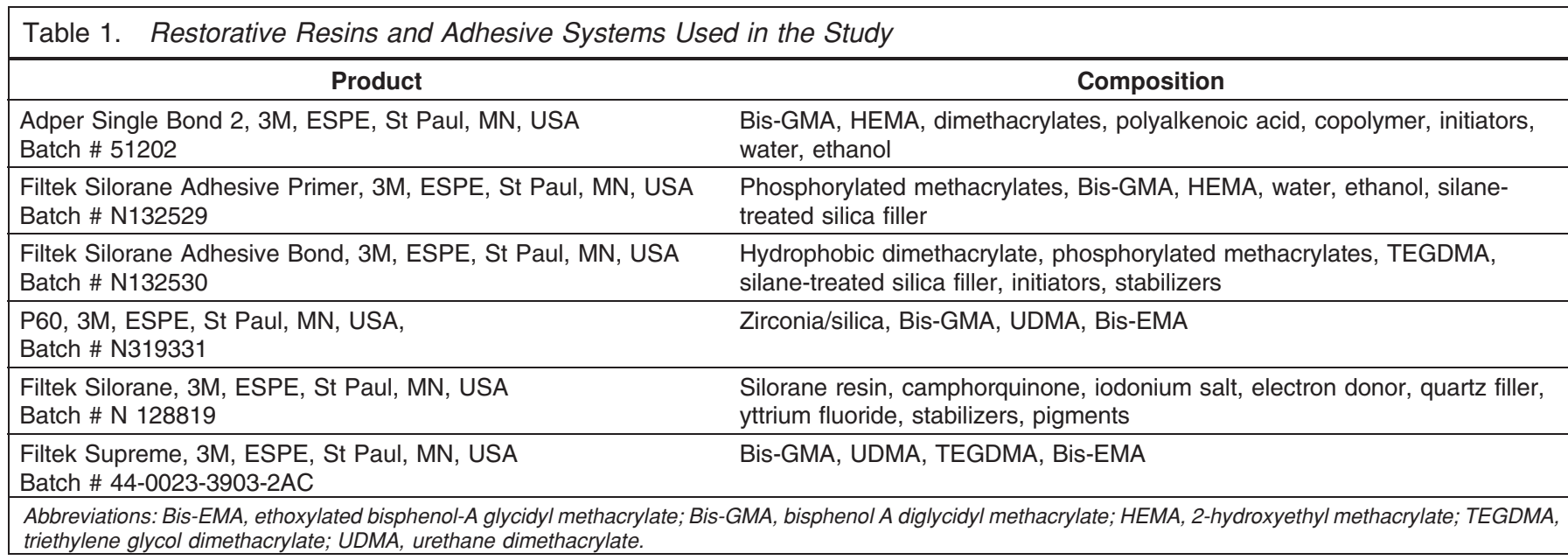




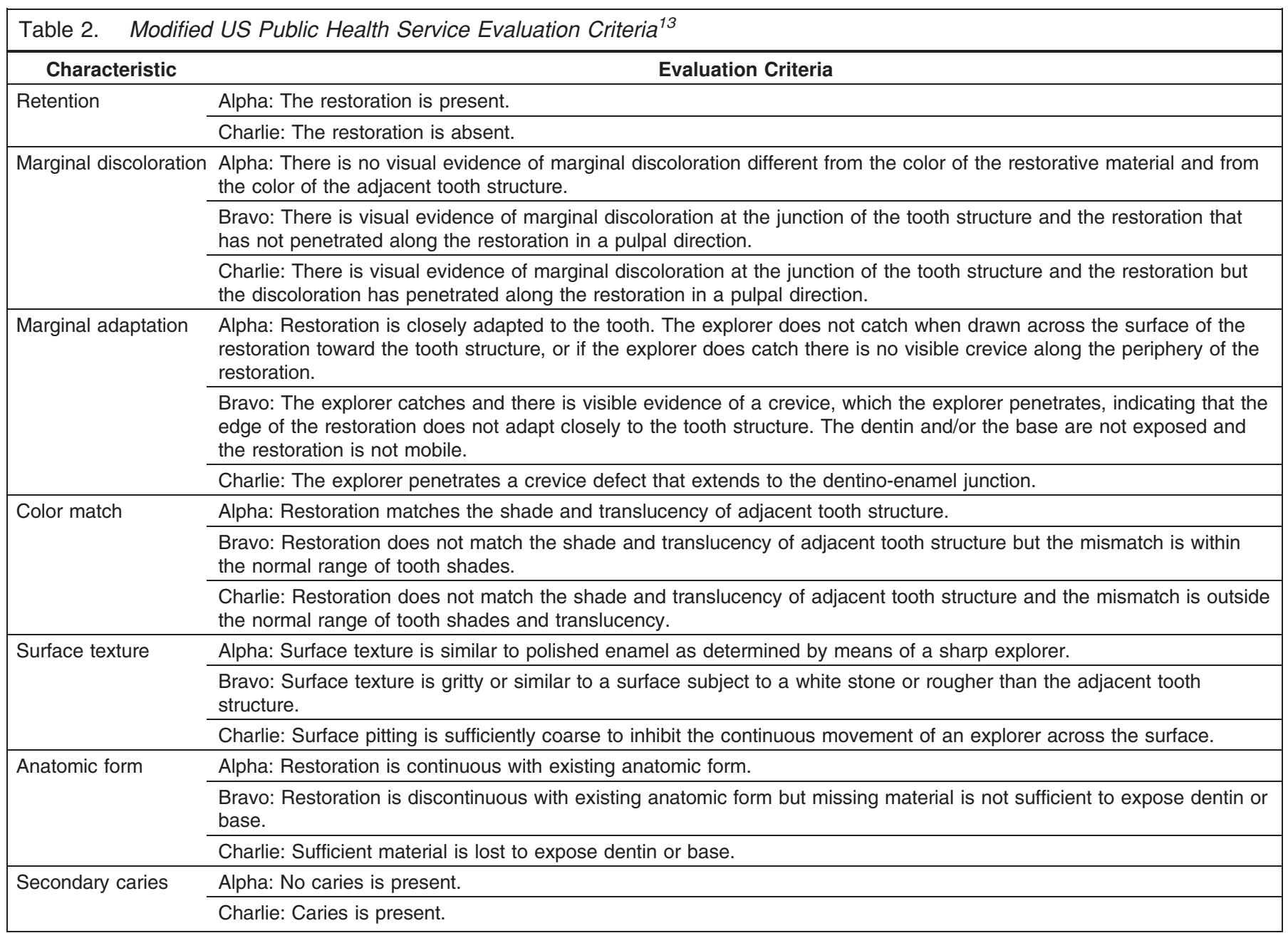

\section{Statistical Analysis}

The three restorative materials for each category were compared using a $\chi^{2}$ test at a significance level of 0.05. Cochran's Q test was used to compare the changes across the five time points for each restorative material. McNemar's test followed by Bonferroni adjustment was used when significant differences were found.

\section{RESULTS}

Three restorations were placed in each subject, resulting in a total of 84 restorations in 28 patients. The recall rate was $100 \%$ at one year. At two years, one patient had moved away and three restorations could not be evaluated (recall rate $=96.4 \%$ ). At the end of three years, 20 patients (60 of 84 restorations) were evaluated (recall rate $=71.4 \%$ ).

Table 3 presents the data for retention, marginal discoloration, marginal adaptation, color match, and surface texture. As no loss of restorations occurred, a
$100 \%$ retention rate was recorded for all three restoratives at the three-year recall. At the sixmonth recall, no statistically significant differences were found between the restorative materials for any of the evaluation criteria tested $(p>0.05)$.

There were no significant differences among the restorative groups in terms of marginal discoloration. After three years, 10 Filtek Silorane restorations, 9 Filtek Supreme restorations, and 8 P60 restorations received Bravo scores. These discolorations occurred at the enamel margin.

For marginal adaptation, there was a significant difference only between P60 and Filtek Silorane at the end of one year. At the two-year recall, Filtek Silorane showed worse marginal adaptation than P60 and Filtek Supreme. Eleven restorations from Filtek Silorane, five from Filtek Supreme, and only three from $\mathrm{P} 60$ received Bravo scores for marginal adaptation at the three-year recall; the difference between Filtek Silorane and P60 was significant $(p<0.05)$. 
With regard to color match, two teeth, one restored with P60 and the other with Filtek Silorane, received Bravo scores at the end of two years and three years. All restorations from the Filtek Supreme group showed Alpha scores.

In terms of surface texture, only one restoration from the P60 group and one from the Filtek Supreme group received Bravo scores, whereas all restorations from the Filtek Silorane group received Alpha scores at the end of three years. No significant differences were observed in color match or surface texture between the restorative materials. All the restorations were scored as Alpha for anatomic form. None of the teeth showed secondary caries or pulp inflammation. None of the subjects reported postoperative sensitivity.

The results for intragroup comparisons between baseline and each evaluation period were as follows. Regarding marginal discoloration, there was only a significant difference between baseline and threeyear results for P60. For Filtek Silorane, statistically significant differences were observed in marginal discoloration between baseline vs two years, baseline vs three years, and six months vs three years. For Filtek Supreme, significant differences were only observed between baseline vs three years and six months vs three years.

In marginal adaptation, there were no statistical differences for any evaluation-period comparisons for P60 and Filtek Supreme. Marginal adaptation significantly worsened over time compared to baseline for Filtek Silorane (baseline vs one year, two years, and three years; and six months vs two years and three years $)(p<0.05)$.

\section{DISCUSSION}

A recent innovation in posterior resin restoratives is the introduction of silorane restorative resin. Many in vitro studies have been published regarding silorane's performance. In a recent in vitro study, the bonding effectiveness and marginal integrity of a silorane restorative system to dentin were compared with those of a methacrylate-based one, P60/Adper Easy Bond. No difference was noted between the silorane-based system and the methacrylate-based system. ${ }^{14}$ The durability of the bond to dentin of the new silorane-based bonding agent, Filtek Silorane System Adhesive, was found to be as good as that of the methacrylate-based composite resin-bonding agent, Clearfil SE Bond. ${ }^{15}$ Similar results were obtained in a study that compared the microtensile bond strength of methacrylate resin systems to a silorane-based restorative system on dentin. The silorane-based system performed similarly to that of methacrylate-based materials on dentin. ${ }^{16}$ On the other hand, in another in vitro study evaluating the marginal adaptation of a methacrylate-based composite and a silorane-based composite, the siloranebased composite exhibited significantly lower shrinkage forces and better marginal adaptation than did the methacrylate-based composite, Filtek Supreme XT. ${ }^{17}$ Although the results of those in vitro studies can help to predict clinical effectiveness of restorative resins, the best way is to conduct clinical trials. In the present study, the three-year clinical performances of nanofilled, packable, and silorane restorative resins were compared.

In terms of marginal adaptation, the results of this clinical study do not appear to confirm the hypothesis that a low-shrinkage restorative resin could show better performance than packable and nanofilled resin composite. Eleven restorations from the silorane group received Bravo scores for marginal adaption at the end of three years. However, significant differences were only seen between P60 and silorane at the end of three years. The reason is probably related to the adhesive system used. Although Filtek Supreme and P60 restorations were performed with the use of an etch-and-rinse adhesive, Adper Single Bond 2, silorane was used with its respective self-etch adhesive system. With a $\mathrm{pH}$ of about 2.7, Silorane System Adhesive Self-Etch Primer provides rather ultra-mild etching and demineralization of the tooth structure and, therefore, might produce less pronounced etching patterns than those achieved with phosphoric acid etching. ${ }^{18}$ Baracco and others ${ }^{10}$ evaluated the oneyear clinical performance in Class I and II cavities of three different restoratives: Filtek Silorane with its respective adhesive and Filtek Z250 used either with an etch-and-rinse adhesive or a two-step self-etch adhesive. Although Filtek Silorane showed acceptable clinical performance, these restorations had no advantage over those with methacrylate-based composite combined with etch-and-rinse adhesive. Moreover, Silorane restorations tended to degrade in terms of marginal adaptation compared with baseline values. In their two-year follow-up, the three restorative systems showed statistically similar clinical performances. ${ }^{12}$ The same results were obtained in the present study. Although there were no differences between baseline and three-year recall results in terms of marginal adaptation in P60 and Filtek Silorane restorations, silorane restorations worsened over three years. 


\begin{tabular}{|c|c|c|c|c|c|c|c|c|c|}
\hline \multirow[t]{2}{*}{ Parameter Rating } & \multicolumn{3}{|c|}{ Baseline } & \multicolumn{3}{|c|}{6 Mo } & \multicolumn{3}{|c|}{$1 \mathrm{Y}$} \\
\hline & Filtek P60 & $\begin{array}{c}\text { Filtek } \\
\text { Silorane }\end{array}$ & $\begin{array}{c}\text { Filtek } \\
\text { Supreme }\end{array}$ & Filtek P60 & $\begin{array}{c}\text { Filtek } \\
\text { Silorane }\end{array}$ & $\begin{array}{c}\text { Filtek } \\
\text { Supreme }\end{array}$ & Filtek P60 & $\begin{array}{c}\text { Filtek } \\
\text { Silorane }\end{array}$ & $\begin{array}{c}\text { Filtek } \\
\text { Supreme }\end{array}$ \\
\hline \multicolumn{10}{|l|}{ Retention } \\
\hline Alpha & $28 / 28(100)$ & $28 / 28(100)$ & $28 / 28(100)$ & $28 / 28(100)$ & $28 / 28(100)$ & $28 / 28(100)$ & $28 / 28(100)$ & $28 / 28(100)$ & $28 / 28(100)$ \\
\hline \multicolumn{10}{|c|}{ Marginal discoloration } \\
\hline Bravo & $28 / 0 \quad(0)$ & $28 / 0 \quad(0)$ & $28 / 0 \quad(0)$ & $28 / 1 \quad(4)$ & $28 / 2 \quad(7)$ & $28 / 1 \quad(4)$ & $28 / 3 \quad(11)$ & $28 / 5 \quad(18)$ & $28 / 4 \quad(14)$ \\
\hline \multicolumn{10}{|c|}{ Marginal adaptation } \\
\hline Alpha & $28 / 28(100)$ & $28 / 28(100)$ & $28 / 28(100)$ & $28 / 27(96)$ & $28 / 24(86)$ & $28 / 26(93)$ & $28 / 27(96)$ & $28 / 19(68)$ & $28 / 25(89)$ \\
\hline Bravo & $28 / 0 \quad(0)$ & $28 / 0 \quad(0)$ & $28 / 0 \quad(0)$ & $28 / 1 \quad(4)$ & $28 / 4 \quad(14)$ & $28 / 2 \quad(7)$ & $28 / 1 \quad(4)$ & $28 / 9 \quad(32)$ & $28 / 3 \quad(11)$ \\
\hline \multicolumn{10}{|l|}{ Color match } \\
\hline Bravo & $28 / 0 \quad(0)$ & $28 / 0 \quad(0)$ & $28 / 0 \quad(0)$ & $28 / 0 \quad(0)$ & $28 / 0 \quad(0)$ & $28 / 0 \quad(0)$ & $28 / 1 \quad(4)$ & $28 / 0 \quad(0)$ & $28 / 0 \quad(0)$ \\
\hline
\end{tabular}

On the other hand, Burke and others ${ }^{8}$ evaluated the clinical performance of 100 restorations (30 Class I and 70 Class II) placed with Filtek Silorane. Satisfactory clinical performance was reported with a rate of $84 \%$ optimal for marginal integrity and $77 \%$ for marginal discoloration at the end of two years. ${ }^{8}$ At the two-year and three-year recalls, respectively, Filtek Silorane received 73\% and 50\% Alpha scores in marginal discoloration and 56\% and 45\% Alpha scores in terms of marginal adaptation.

In a short-term clinical study, Goncalves and others ${ }^{11}$ compared the proximal contact of a silorane-based resin composite with a conventional methacrylate-based resin composite, P60, in Class II restorations. Both restorative resins gave satisfactory results. However, these results were obtained after six months of clinical service. The follow-up period seems very short and differences could develop over longer periods of use; therefore, these results cannot be directly compared with our study's results.

Only three P60 restorations showed Bravo scores in terms of marginal adaptation at the end of three years. The superior behavior of P60 has been demonstrated in a study conducted by Kiremitci and others ${ }^{19}$ In their clinical study, the clinical performance of P60 was acceptable after six years of service. Regarding marginal adaptation and discoloration, $95 \%$ and $91 \%$ of $\mathrm{P} 60$ restorations, respectively, received an Alpha rating. In another study, the clinical performance of two adhesive restorative systems (Single Bond/Filtek P-60 and Single Bond/
Filtek Z-250) was assessed in posterior teeth. ${ }^{20}$ Marginal integrity for P-60 was scored as 94.3\% and $91.4 \%$ Alpha at six and 12 months, respectively. All restorations were found to be clinically satisfactory.

In a recent study, the clinical performance of Filtek Supreme used with either etch-and-rinse or self-etch adhesive in Class I cavities was evaluated. ${ }^{21}$ At the end of one year, composite resin restorations using either adhesive system showed equally satisfactory results. In another study, the performance of Filtek Supreme was found to be satisfactory even when used with a self-etch adhesive, Adper Prompt-L-Pop, over 15 months of clinical service. $^{22}$

In a one-year clinical study, the performance of a nanofilled resin composite for posterior restorations was compared with that of two microhybrid composites and one packable composite. ${ }^{23}$ Similar to our findings, the nanofilled resin composite showed a performance similar to that of the other packable and microhybrid resin composites. Sadeghi and others ${ }^{24}$ evaluated the clinical performance of microhybrid, packable, and nanofilled resin composite restorations placed in Class I cavities in molar teeth and found no difference between the restorations over 18 months.

In the present study, no significant differences were found between the restorative materials in terms of marginal discoloration. It is known that if the bond between the resin and tooth structure is 


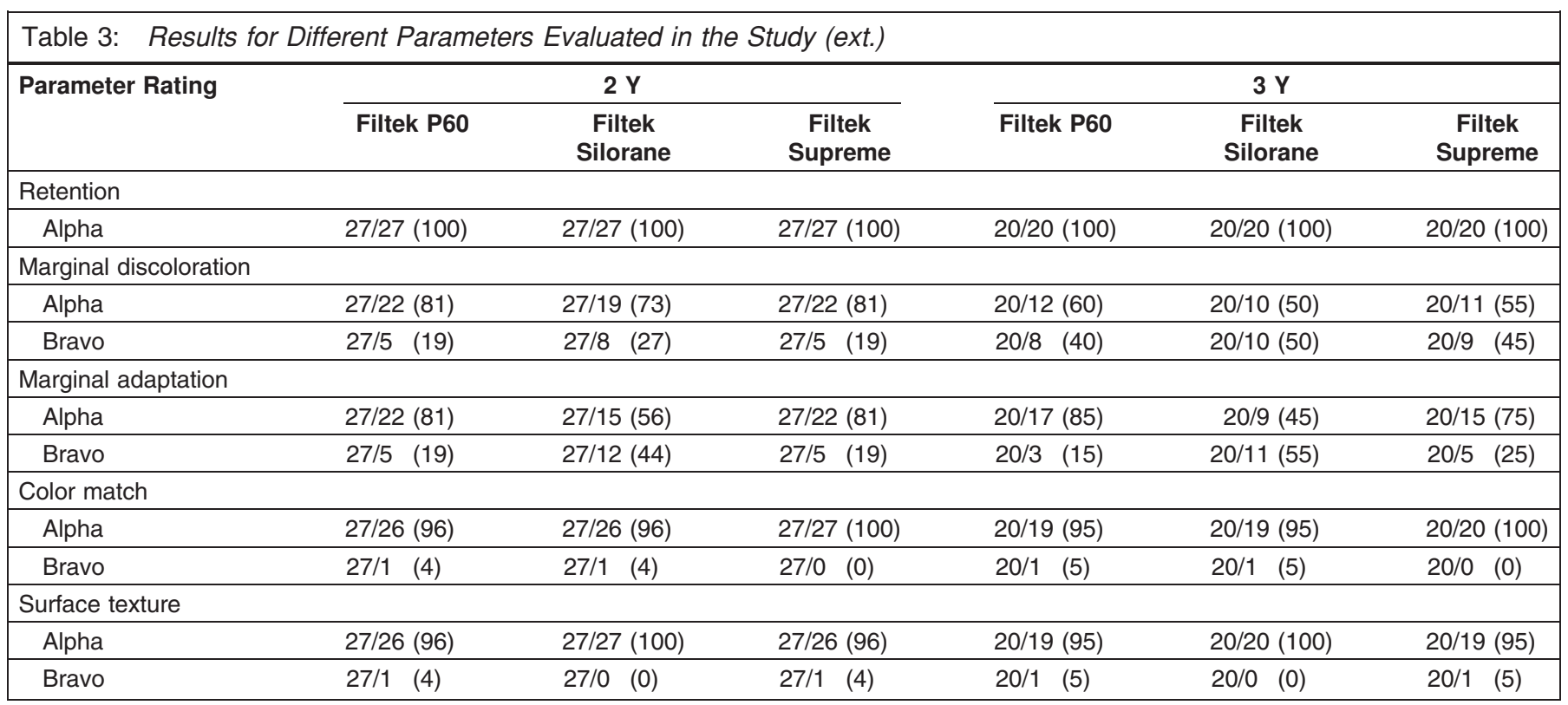

less than the polymerization shrinkage force, microleakage and marginal failure will occur. ${ }^{1}$ Marginal discoloration is generally related to microleakage. All the restorations showed quite similar results in terms of marginal discoloration despite having different thermal expansion coefficients and polymerization shrinkage rates. These discolorations were superficial and localized. Although silorane system adhesive is self-etching, chemical bonding to the hydroxyapatite crystals occurs with the silorane's primer. ${ }^{25}$ Moreover, the primer and adhesive resin of this system are separately cured, which means a twofold bonding layer ${ }^{25}$ that might also act as an elastic buffer. ${ }^{26}$ This might be a reason for the similar results obtained with nanofilled and packable resin composite restorations.

Postoperative sensitivity is one of the most common complaints of patients with posterior resin restoratives. Class I resin composite restorations are more prone to postoperative sensitivity and marginal failure because of the higher cavity configuration factor $(\mathrm{C}=5)$. However, in the present study, none of the subjects reported postoperative sensitivity. This might have been related to the insertion of resin. As incremental placement was used, polymerization shrinkage stress might have been decreased. Other evaluation criteria, such as anatomic form, color match, and surface texture remained optimal over the three-year period.

In the present study, no restoration loss was observed during the three years of clinical functioning. All the restorative resins presented an almost excellent clinical performance with a $100 \%$ retention rate and no statistically significant difference between them except in marginal adaptation. However, this clinical trial was conducted in Class I cavities with a retentive cavity shape and margins lying on enamel. Different cavity types, such as Class II extending to the dentin, might have yielded significant differences. Therefore, further clinical studies addressing different cavity types should be conducted.

\section{Conclusion}

Within the limitations of this clinical study, it is concluded that low shrinkage silorane-based restorative, packable, and nanofilled composite resin showed similar clinical results with the exception of packable resin composite, which showed a better performance in terms of marginal adaptation.

\section{Acknowledgements}

The authors thank 3M/ESPE for providing materials for this study and Sevilay Karahan for assistance with statistical analysis.

\section{Conflict of Interest}

The authors of this manuscript certify that they have no proprietary, financial, or other personal interest of any nature or kind in any product, service, and/or company that is presented in this article.

(Accepted 2 October 2013)

\section{References}

1. Leinfelder KF, Bayne SC, \& Swift EJ Jr (1999) Packable composites: overview and technical considerations Journal of Esthetic Dentistry 11(5) 234-249. 
2. Choi KK, Ferracane JL, Hilton TJ, \& Charlton D (2000) Properties of packable dental composites Journal of Esthetic Dentistry 12(4) 216-226.

3. Reis AF, Giannini M, Lovadino JR, \& Ambrosano GM (2003) Effects of various finishing systems on the surface roughness and staining susceptibility of packable composite resins Dental Materials 19(1) 12-18.

4. Borges AB, Marsilio AL, Pagani C, \& Rodrigues JR (2004) Surface roughness of packable composite resins polished with various systems Journal of Esthetic and Restorative Dentistry 16(1) 42-47.

5. Mitra SB, Wu D, \& Holmes BN (2003) An application of nanotechnology in advanced dental materials Journal of the American Dental Association 134(10) 1382-1390.

6. Karaman E, \& Ozgunaltay G (2013) Cuspal deflection in premolar teeth restored using current composite resins with and without resin-modified glass ionomer liner Operative Dentistry 38(3) 282-289.

7. 3M ESPE (2007) Technical information for Filtek Silorane. Retrieved online May 7, 2013 from: http://www. odontoiatra.it/files/tecniche_463_11.pdf.

8. Burke FJ, Crisp RJ, James A, Mackenzie L, Pal A, Sands P, Thompson O, \& Palin WM (2011) Two- year clinical evaluation of a low-shrink resin composite material in UK general dental practices Dental Materials 27(7) 622-630.

9. Schmidt M, Kirkevang LL, Horsted-Bindslev P, \& Poulsen S (2011) Marginal adaptation of a low-shrinkage silorane-based composite: 1-year randomized clinical trial Clinical Oral Investigation 15(2) 291-295.

10. Baracco B, Perdigao J, Cabrera E, Giraldez I, \& Ceballos L (2012) Clinical evaluation of a low-shrinkage composite in posterior restorations: one-year results Operative Dentistry 37(2) 117-129.

11. Goncalves FS, Castro CD, Bueno AC, Freitas AB, Moreira AN, \& Magalhaes CS (2012) The short-term clinical performance of a silorane-based resin composite in the proximal contacts of Class II restorations Journal of Contemporary Dental Practice 13(3) 251-256.

12. Baracco B, Perdigao J, Cabrera E, \& Ceballos L (2013) Two-year clinical performance of a low-shrinkage composite in posterior restorations Operative Dentistry $\mathbf{3 8 ( 6 )}$ 591-600.

13. Cvar JF, \& Ryge G (1971) Criteria for the clinical evaluation of dental restorative materials US Public Health Services Publication, No 790-244 Government Printing Office, San Francisco.

14. Santos PJ, Silva MS, Alonso RC, \& D'Alpino PH (2013) Hydrolytic degradation of silorane- and methacrylatebased composite restorations: evaluation of push-out strength and marginal adaptation Acta Odontologia Scandinavia 71(5) 1273-1279.
15. Giacobbi MF, \& Vandewalle KS (2012) Microtensile bond strength of a new silorane-based composite resin adhesive General Dentistry 60(3) e148-e152.

16. Sampaio RK, Wang L, Carvalho RV, Garcia EJ, Andrade AM, Klein-Junior CA, Grande RH, \& Moura SK (2013) Six-month evaluation of a resin/dentin interface created by methacrylate and silorane-based materials Journal of Applied Oral Science 21(1) 80-84.

17. Gregor L, Bortolotto T, Feilzer AJ, \& Krejci I (2013) Shrinkage kinetics of a methacrylate- and a siloranebased resin composite: effect on marginal integrity Journal of Adhesive Dentistry 15(3) 245-250.

18. Sarr M, Kane AW, Vreven J, Mine A, Van Landuyt KL, Peumans M, Lambrechts P, Van Meerbeek B, \& De Munck J (2010) Microtensile bond strength and interfacial characterization of 11 contemporary adhesives bonded to bur-cut dentin Operative Dentistry 35(1) 94-104.

19. Kiremitci A, Alpaslan T, \& Gurgan S (2009) Six-year clinical evaluation of packable composite restorations Operative Dentistry 34(1) 11-17.

20. Gianordoli Neto R, Santiago SL, Mendonca JS, Passos VF, Lauris JR, \& Navarro MF (2008) One year clinical evaluation of two different types of composite resins in posterior teeth Journal of Contemporary Dental Practice 9(4) 26-33.

21. Sundfeld R, Scatolin R, Oliveira F, Machado L, Alexandre $R$, \& Sundefeld M (2012) One-year clinical evaluation of composite restorations in posterior teeth: effect of adhesive systems Operative Dentistry 37(6) E1-E8.

22. Manchorova NA, Vladimirov SB, Donencheva ZK, Drashkovich IS, Manolov SK, \& Todorova VI (2008) Clinical evaluation of restorations with self-etch adhesive and nanofilled composite in Class I and Class II cavities Folia Medica (Plovdiv) 50(1) 46-52.

23. Dresch W, Volpato S, Gomes JC, Ribeiro NR, Reis A, \& Loguercio AD (2006) Clinical evaluation of a nanofilled composite in posterior teeth: 12-month results Operative Dentistry 31(4) 409-417.

24. Sadeghi M, Lynch CD, \& Shahamat N (2010) Eighteenmonth clinical evaluation of microhybrid, packable and nanofilled resin composites in Class I restorations Journal of Oral Rehabilitation 37(7) 532-537.

25. Mine A, De Munck J, Van Ende A, Cardoso MV, Kuboki T, Yoshida Y, \& Van Meerbeek B (2010) TEM characterization of a silorane composite bonded to enamel/dentin Dental Materials 26(6) 524-532.

26. Van Meerbeek B, Willems G, Celis JP, Roos JR, Braem M, Lambrechts P, \& Vanherle G (1993) Assessment by nanoindentation of the hardness and elasticity of the resindentin bonding area Journal of Dental Research 72(10) 1434-1442. 